\title{
SIADH-related hyponatremia in hospital day care units: clinical experience and management with tolvaptan
}

\author{
Ramón De las Peñas ${ }^{1} \cdot$ Santiago Ponce $^{2} \cdot$ Fernando Henao $^{3} \cdot$ Carlos Camps Herrero $^{4}$. \\ Enric Carcereny ${ }^{5}$ - Yolanda Escobar Álvarez ${ }^{6}$. César A. Rodríguez ${ }^{7}$. \\ Juan Antonio Virizuela ${ }^{3} \cdot$ Rafael López López $^{8}$
}

Received: 12 May 2015 / Accepted: 14 September 2015 /Published online: 2 October 2015

(C) The Author(s) 2015. This article is published with open access at Springerlink.com

\begin{abstract}
Hyponatremia ( $\mathrm{Na} 135 \mathrm{mmol} / \mathrm{l}$ ) is the most frequent electrolyte disorder in clinical practice, and the syndrome of inappropriate antidiuretic hormone secretion (SIADH) is the commonest cause of hyponatremia in cancer patients. Correcting hyponatremia in these patients can reduce morbidity and mortality, increase the response to anti-cancer agents, and help reduce hospital length of stay and costs. Tolvaptan is an oral medication used to treat SIADH-related hyponatremia patients that needs to be initiated at hospital so patients can have their serum sodium monitored. If tolvaptan could be initiated in hospital day care units (DCUs),
\end{abstract}

Camps, Carcereny, Escobar, Rodriquez and Virizuela contributed equally to this work.

Ramón De las Peñas

ramon.delaspenas@hospital2000.net

1 Medical Oncology Department, Consorcio Hospitalario Provincial de Castellón, Castellón, Spain

2 Medical Oncology Department, Hospital 12 de Octubre, Madrid, Spain

3 Medical Oncology Department, Hospital Universitario Virgen de la Macarena, Seville, Spain

4 Medical Oncology Department, Consorcio Hospital General Universitario de Valencia, University of Valencia, Valencia, Spain

5 Medical Oncology Department, Institut Catalàd'Oncologia-Badalona, Hospital Germans Trias i Pujol, Barcelona, Spain

6 Medical Oncology Service, Hospital General Universitario Gregorio Marañón, Madrid, Spain

7 Medical Oncology Department, Hospital Universitario de Salamanca-IBSAL, Salamanca, Spain

8 Medical Oncology Department, Hospital Clínico Universitario, Santiago de Compostela, Spain performing the same tests, hospitalization could be avoided, quality of life improved, and costs reduced. This is the first publication where a panel of oncologists are sharing their experience and making some recommendations with the use of tolvaptan to treat SIADH-related hyponatremia in DCU after collecting and examining 35 clinical cases with these type of patients. The conclusion from this retrospective observational analysis is that the use of tolvaptan in DCU is safe and effective in the therapeutic management of SIADH-related hyponatremia.

Keywords Antidiuretic hormone receptor antagonists · Day care units $\cdot$ Hyponatremia $\cdot$ Inappropriate ADH syndrome . Tolvaptan

\section{Introduction}

Hyponatremia $(\mathrm{Na}<135 \mathrm{mmol} / \mathrm{l})$ is the most common electrolyte disorder in clinical practice, affecting around 15-28\% of hospitalized patients [1] and occurring in up to $44 \%$ in certain cancers, such as small cell lung cancer (SCLC) [2]. The most frequent cause of hyponatremia in the hospital setting is the syndrome of inappropriate antidiuretic hormone secretion (SIADH) [3]. SIADH is also the commonest cause of hyponatremia in cancer patients, representing around $30 \%$ of the total [4].

Hyponatremia is associated with high morbidity and mortality $[5,6]$ and poorer response to anti-cancer agents [2]. It causes longer hospital length of stay and increases medical costs [6-8].

Tolvaptan is an oral treatment indicated for adult patients with hyponatremia secondary to SIADH [9]. This is the first publication about the use of tolvaptan in hospital day care unit (DCU). 


\section{Definition, classification, and diagnosis of hyponatremia}

Hyponatremia is defined as a serum sodium concentration below $135 \mathrm{mmol} / \mathrm{l}$. It is considered "mild" if values are between 130 and $135 \mathrm{mmol} / \mathrm{l}$, "moderate" if between 120 and $129 \mathrm{mmol} / \mathrm{l}$, and "severe" if below $120 \mathrm{mmol} / \mathrm{l}$. As for the symptoms, it can be classified as mild, moderate, or severe according to their intensity. Hyponatremia is considered "acute" if developed in less than $48 \mathrm{~h}$ or "chronic" if developed over a longer period of time [10]. Also, it can be classified as iso-osmolar, hyperosmolar, and hypotonic or hypoosmolar [10]. Hypotonic hyponatremia is most frequently encountered in clinical practice (60\% of all cases), especially in cancer patients, and the commonest cause of hypotonic hyponatremia with euvolemia is SIADH [10].

The differential diagnosis of hyponatremia can be performed with an exhaustive anamnesis, physical examination assessing the extracellular fluid volume status, and simple blood and urine laboratory tests $[2,11]$.

\section{SIADH-related hyponatremia}

SIADH is commonly caused by cancers, certain medications, central nervous system (CNS) disorders, pulmonary diseases, or other causes (AIDS, idiopathic, etc.) [12].

In cancer patients, SIADH is a paraneoplastic syndrome associated with a wide range of tumors but about $75 \%$ of all cases are encountered in SCLC [13]. SIADH may be a result of ectopic arginine vasopressin (AVP) production by tumor cells or a result of stimulation of AVP secretion or potentiation of AVP effects by some anti-cancer drugs as well as by other factors like diarrhea or vomiting (Table 1) $[2,11]$.
Before turning the diagnosis of SIADH, other clinical entities need to be excluded like hypothyroidism, hypopituitarism, severe emotional or physical stress, and medications that stimulate AVP release [14]. Several consensus documents have established the diagnosis criteria of SIADH in cancer patients (Table 2) [10].

\section{Clinical consequences of hyponatremia due to SIADH}

Hypotonic hyponatremia causes cerebral oedema, intracranial hypertension, and risk of brain injury $[15,16]$. Patients with mild hyponatremia are traditionally asymptomatic. At serum concentrations between 125 and $130 \mathrm{mmol} / \mathrm{l}$, anorexia, nausea, vomiting, and abdominal pain may develop. As serum sodium concentration falls, agitation, confusion, hallucinations, incontinence, and other neurological symptoms predominate. Hyponatremia below $115 \mathrm{mmol} / 1$ may induce serious neurological squeals and constitutes a medical emergency [17].

\section{Treatment of SIADH-related hyponatremia in cancer patients}

Untreated acute hyponatremia can cause substantial morbidity and mortality as a result of osmotically induced cerebral edema, and excessively rapid correction can cause severe neurologic impairment and death as a result of osmotic demyelination [16]. Some recently published guidelines have reviewed the management of SIADH in cancer patients (Fig. 1) [10, 18].

Table 1 The causes of SIADH in cancer patients

\begin{tabular}{|c|c|c|}
\hline Cancer & $\begin{array}{l}\text { Drugs known to cause hyponatremia by affecting AVP } \\
\text { production/action }\end{array}$ & Lung diseases \\
\hline $\begin{array}{l}\text { SCLC } \\
\text { Gastrointestinal cancer }\end{array}$ & Mechanism: increased hypothalamic AVP production & $\begin{array}{l}\text { Infections: pneumonia (bacterial or viral), } \\
\text { tuberculosis }\end{array}$ \\
\hline $\begin{array}{l}\text { Pancreas adenocarcinoma } \\
\text { Hematological cancers }\end{array}$ & $\begin{array}{l}\text { Anti-cancer agents: Vinca alkaloids, platinum compounds, } \\
\text { alkylating agents, and others }\end{array}$ & \\
\hline Sarcomas & $\begin{array}{l}\text { Palliative medications: opioid analgesics, antidepressants, } \\
\text { antipsychotics, and antiepileptics }\end{array}$ & Respiratory acute failure \\
\hline CNS (primary and metastatic cancer) & Mechanism: potentiation AVP actions & \\
\hline Prostate adenocarcinoma & Anti-cancer agents: alkylating agents & \\
\hline $\begin{array}{l}\text { Urothelial cancer } \\
\text { Head and neck squamous cancer }\end{array}$ & $\begin{array}{l}\text { Palliative medications: nonsteroidal anti-inflammatory } \\
\text { drugs, antiepileptic and antidiabetic agents }\end{array}$ & \\
\hline \multicolumn{3}{|l|}{ Endometrial adenocarcinoma } \\
\hline \multicolumn{3}{|l|}{ Thymoma } \\
\hline Neuroendocrine tumors & & \\
\hline
\end{tabular}

$A V P$ arginine vasopressin, $C N S$ central nervous system, $S C L C$ small cell lung cancer

Table adapted from Alcázar et al., FMC Oncología, March 2013, and Castillo et al., The Oncologist, 2012; 17:756-65 
Table 2 SIADH diagnostic criteria

Serum $\mathrm{Na}<135 \mathrm{mmol} / 1$

Plasma osmolarity $<275 \mathrm{mOsm} / \mathrm{kg}$

Urine osmolarity $>100 \mathrm{mOsm} / \mathrm{kg}$

Hypotonic hyponatremia with clinical euvolemia

Absence of signs of hypovolemia (normal eye pressure values, normal venous pressure, no orthostatism)

Absence of signs of hypervolemia (ascites, edemas)

Urinary $\mathrm{Na}>40 \mathrm{mmol} / \mathrm{l}$ (in the presence of dietary sodium)

No hypothyroidism, no adrenal insufficiency (hypocortisolism) or renal failure

No recent diuretic intake

Absence of physiological stimuli for AVP secretion (recent surgery, severe pain AVP secretion stimulating drugs...)

Table adapted from De las Peñas R et al. Clin Trans Oncol. 2014

AVP arginine vasopressin

\section{Treating SIADH in hospital DCU}

Hyponatremia is more common in hospitalized patients than in ambulatory individuals [19] and is independently associated with a higher risk in death, with increased length of stay and cost of care for hospitalized patients [5-8]. Mild hyponatremia occurs in 15 to $22 \%$ of those patients and in approximately $7 \%$ of ambulatory patients; moderate hyponatremia occurs in 1 to $7 \%$ of hospitalized patients [12]. Oncology DCU allow patients to receive specialized care without needing to be hospitalized. Tolvaptan is an oral medication that in spite of being safe, like stated in the summary of product characteristics, it needs to be initiated at hospital so patients can have their serum sodium monitored [9]. If tolvaptan could be initiated in hospital DCU, hospitalization could be avoided, quality of life improved, and costs reduced.

\section{Rationale for the use of tolvaptan in hospital DCU}

\section{Clinical and economic impact of hyponatremia in hospitalized cancer patients}

Several studies and a meta-analysis, with hospitalized oncology patients, show the negative prognostic impact of the presence of hyponatremia in survival, clinical outcomes, and quality of life, and on the other hand, economic data can be extrapolated from studies on non-cancer patients regarding prolongation of hospital stay and increased hospital costs for this reason.
A

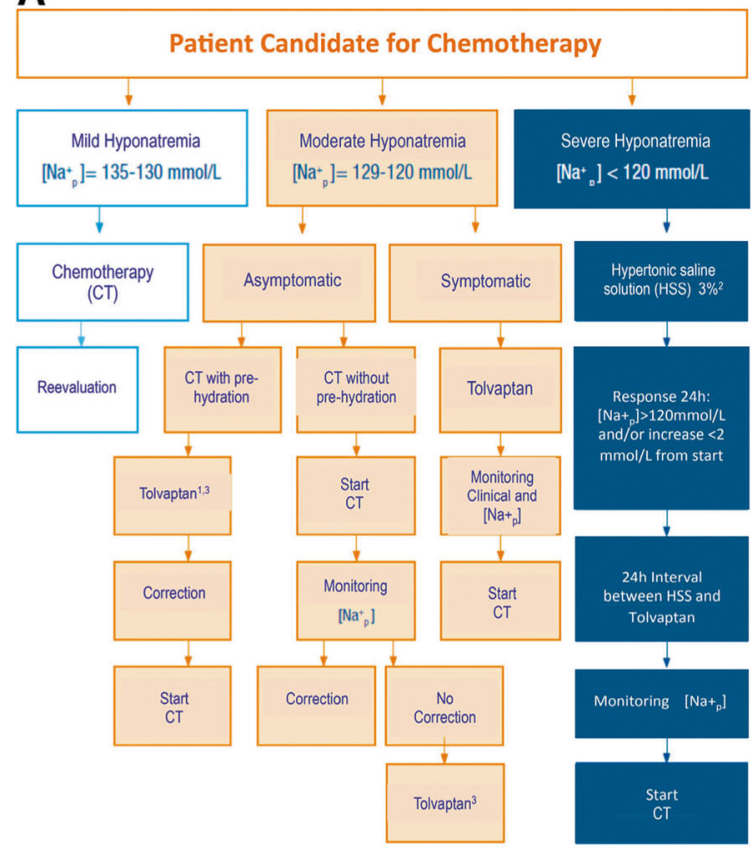

1.- Administer Tolvaptan until improvement of natremia and then start chemotherapy. Can be administered jointly with chemotherapy under strict control of diuresis.

2.- Patient weight $=\mathrm{mL} / \mathrm{h}$ of SS $3 \%$; with control of plasma $\mathrm{Na}+$ every 2 hours at start.

3.- Check Tolvaptan SPC.
B

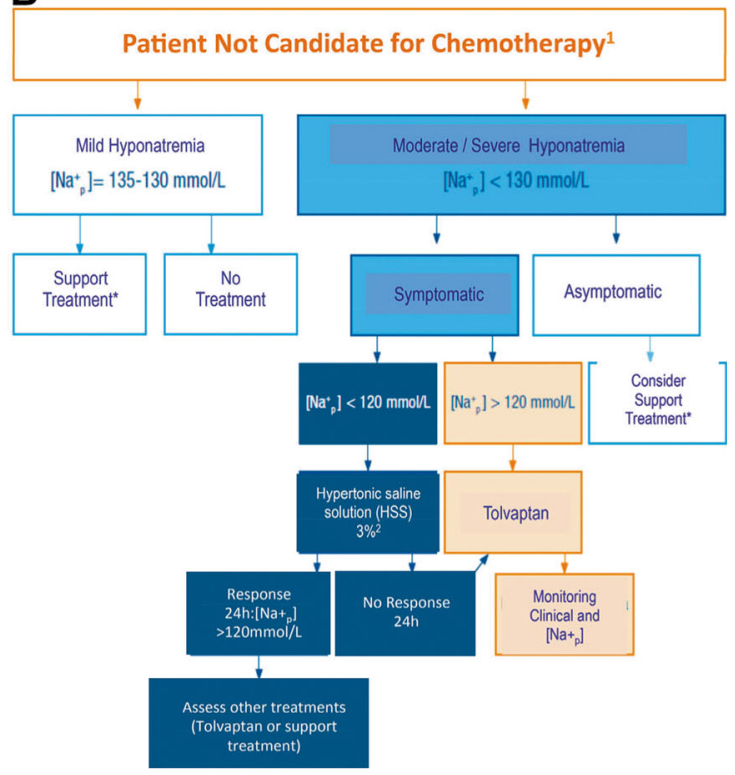

1.- Patients candidates for endocrine, biological, immune therapy ol exclusive symptomatic treatment.

2.- Patient weight $=\mathrm{mL} / \mathrm{h}$ of SS $3 \%$; with control of plasma $\mathrm{Na}+$ eveI 2 hours at start. *Support treatments: water restriction. Salt supplement.

Fig. 1 Treatment algorithm for hyponatremia in cancer patients. De las Peñas et al. Clin Transl Oncol. 2014; 16:1051-9 
Clinical impact on survival, chemotherapy outcomes/patients prognosis, and ECOG score

Hyponatremia is associated with an increase in hospital mortality Several studies in oncology patients showed direct alterations on survival in relation with hyponatremia [11, 20-22]. A low serum sodium level could affect negatively the performance status and the quality of life of cancer patients, and may delay time-critical treatments such as chemotherapy [22]. Even mild hyponatremias have been associated with an increase in mortality in hospitalized patients [23]. In 3357 cancer patients hospitalized at the MD Anderson Cancer Center in 2006 for 3 months, hyponatremia was associated with worst survival during 90 days [24]. An analysis of all adult patients discharged from an academic medical center, during a 7-year period showed that mortality was significantly associated with a serum $\mathrm{Na}^{+}<138 \mathrm{mmol} / 1$ and $\mathrm{Na}^{+}$ $>142 \mathrm{mmol} / \mathrm{l}[25]$.

Patients with hyponatremia who do not fully normalize the serum sodium value have a worse prognosis than those who normalize it Hyponatremia was associated with poorer prognosis in a study with 402 patients treated with carboplatin-etoposide. Interestingly, patients with ectopic production of atrial natriuretic peptide (ANP) are reported to respond poorly to water restriction, and lack of response to water restriction within few days is suggested as screening test for ectopic production of ANP in hyponatremia SCLC patients. The treatment of hyponatremia at the study institution has predominantly been the prescription of water restriction, and in the series only $25 \%$ of the patients with hyponatremia $(<130 \mathrm{mmol} / \mathrm{l})$ had fully normalized serum sodium to values above $135 \mathrm{mmol} / \mathrm{l}$ at the time of the second cycle of chemotherapy 3-4 weeks after the first cycle [20].

Hyponatremia is associated with worsening of serum sodium levels during hospitalization In the Anderson Cancer Center study, hyponatremia was noted in $47 \%$ of the patients (23\% at admission and $24 \%$ was acquired during hospitalization) [24]. Data from an academic medical center in Canada reveal that hyponatremia worsening after admission was observed in $6 \%$ of hospitalizations associated with communityacquired hyponatremia, while $38 \%$ developed during hospitalization [25].

Satisfaction with care in cancer patients treated at hospital DCU is high A study that evaluated the satisfaction with care in cancer patients treated at hospital DCU showed that satisfaction with care in this setting is high and correlation between satisfaction with care and quality of life indicated that these two concerns are complementary [26].
The ECOG performance status score at admission shows a negative correlation with the sodium status A study that checked the effects of hyponatremia among patients with lung carcinoma on their Eastern Cooperative Oncology Group (ECOG) performance showed that the ECOG score at admission shows a negative correlation with the serum sodium status measured on admission among all the patients. The ECOG score correlated with the duration of the patient survival and the response to treatment, as well as their quality of life and co-morbidity. This study concludes that hyponatremia may influence the ECOG performance status score, which serves as an important factor in the prognosis of lung cancer [27].

Economic impact in hospital costs and hospital length of stay and complications

Hyponatremia increases hospital costs A retrospective analysis that evaluated the impact of varying levels of hyponatremia at admission on cost of care in adult hospitalized patients showed median costs of $\$ 16,606$ for moderateto-severe hyponatremia cases, $\$ 14,266$ for mild-to-moderate, and $\$ 13,066$ for normal admissions $(P<0.001)$. Based on an annual admission rate at that institution of approximately 55 , 000 , it was estimated that patients admitted with hyponatremia were associated with an additional \$2.15 million in hospital costs and over 3400 additional bed days [28].

Data from the Integrated HealthCare Information Services National Managed Care Benchmark Database showed that hyponatremia was associated with $99 \%$ higher 6-months costs, and also associated with $108 \%$ higher 1-year medical costs. It was estimated that hyponatremia increased direct medical costs at 6 and 12 months (41. 2 and $45.7 \%$, respectively) [29].

An analysis performed to estimate the annual cost of hyponatremia in the USA showed that $55-63 \%$ of hyponatremia patients are initially treated as inpatients, $25 \%$ are initially treated in the emergency room, and $13-20 \%$ are treated solely in the office setting. The direct costs of treating hyponatremia in the USA on an annual basis were estimated to range from $\$ 1.6$ to $\$ 3.6$ billion. Hospitalization costs (including readmissions) accounted for approximately $70 \%$ of the total cost of illness [30].

Hyponatremia in cancer patients at admission is associated with longer length of stay and hospital complications The Anderson Cancer Center study showed that the length of stay of patients with hyponatremia was significantly higher than for those with normal sodium levels $(10.2+10.2 \mathrm{vs}$. $5.6+5.0$ days; $P<0.01$ ), and for those mild/moderate hyponatremia cases, the length of stay was also higher (9.9 and $13.0+14.1$ vs. $5.6+5.0$ days) [24].

On the other hand, patients with more severe hyponatremia were also more likely to be admitted to the intensive care unit during the hospital stay (moderate-to-severe hyponatremia, 
$32 \%$; mild-to-moderate hyponatremia, $26 \%$; normal, $22 \%$; $P<0.001)$ [28].

The extrapolation of these data to studies with cancer patients confronts us with the fact that almost $50 \%$ of hospitalized patients have hyponatremia, as we have seen, and its presence prolongs the duration of hospitalization in 2-5 days [28]. A recent study with 105 consecutive cancer patients showed a statistically significant correlation between the length of stay and the detection of hyponatremia both at admission $(P=0.0009)$ and during hospitalization ( $P=0.0001)$. In this study, and according to this length of stay, a patient with moderate hyponatremia cost $128 \%$ more than a normonatremic patient [21].

\section{Advantages for the use of tolvaptan in DCU}

DCU are specialized places where patients can have all the controls needed after the first administration of tolvaptan so, taking into account the mentioned clinical and economic impact of hyponatremia in hospitalized cancer patients, we can presume that the ambulatory early correction with tolvaptan in DCU can:

- Facilitate the administration of chemotherapy cycles in a timely manner, which could probably improve the tumor disease and increase patient's survival.

- Ease the prompt correction of serum sodium levels, for a quick start of chemotherapy, which would presumably have a positive impact in the patients' prognosis.

- Minimize the risk of hyponatremia getting worst during hospitalization, when possible.

- Make the patient's treatment compatible with their daily activities and allow these patients to be closer to their families.

- Have a positive impact in the quality of life of oncology patients with hyponatremia.

- Reduce costs originated from hospitalization as well as decrease the emergencies which are the usual route of entry for these patients to hospital.

- Prevent hospital admission, in selected patients, with the associated hospitalization risks like prolonged length of stay and admission to intensive care units helping reduce hospital costs as well as health complications for the patient.

\section{Management and recommendations for the use of tolvaptan to treat SIADH in hospital DCU}

\section{Clinical experience based on clinical cases with the use of tolvaptan in hospital DCU}

Active treatment of SIADH-related hyponatremia must be started as soon as possible to avoid delays in the patients' oncologic treatment [31], and also because in a significant number of cases, the hyponatremia origin is paraneoplastic and the tumor treatment itself becomes a key factor for the correction of the natremia [11].

While in severe, very symptomatic situations, hypertonic saline solution is considered the standard of care $[10,11,31]$ in those cases of moderate hyponatremia, where a controlled increase of the natremia is desired, the first line treatment would be hydric restriction or tolpalvan administration [11]. In particular, hydric restriction may not be possible in the oncologic population because these patients require high hydration for their intravenous treatment administration. Moreover, the natremia correction rate is often inadequate and has a low treatment adherence [11]. Tolvaptan meets the requirements of rapidity, efficacy, safety, and adherence, so it can be considered a good treatment choice, especially in those patients in whom the treatment of the malignancy that is causing the SIADH requires an immediate start of chemotherapy that cannot be administered without adequate hydration volumes $[11,13]$ or in malnourished patients, for whom liquid restriction would worsen their nutritional state [31].

Tolvaptan is usually initiated in hospital due to the need for a dose titration phase with close monitoring of serum sodium and volume status 4-6 h after its administration [9]. However, since this type of control can be done at DCU, these units could help avoid hospitalization in some selected cancer patients with hyponatremia, with all the advantages described previously.

To evaluate this retrospectively, a series of 35 clinical cases were collected from cancer patients receiving treatment with tolvaptan in 3 hospital DCU in Spain [Hospital Provincial de Castellón, Castellón (from September 2011 to May 2014); Hospital Doce de Octubre, Madrid (from March 2013 to September 2014); and Hospital Virgen de la Macarena, Sevilla (from August 2011 to December 2014)] (Table 3).

In all cases, it was suspected a paraneoplastic-related SIADH, and $63 \%$ of the patients underwent treatments that could exacerbate the hyponatremia (18 with opioids, 5 with diuretics, and 3 with antidepressants). Furthermore, $74 \%$ of these patients showed symptomatic hyponatremia and 11 of them showed at least two characteristic symptoms of this electrolyte disorder. The most predominant symptomatology was neurologic. Three patients with severe symptoms were initially treated with hypertonic saline solution. After having controlled the acute initial situation in these patients, they underwent hydric restriction together with other eight patients that did not require this initial treatment. Afterwards, when the criteria for natremia correction were not achieved, tolvaptan was used. In the remaining patients that were not candidates for hydric restriction, tolvaptan treatment was initiated directly, following the general guidelines of the treatment algorithm described in Fig. 1. When tolvaptan was used, no significant changes in vital signs described in Fig. 1 were observed.

The results showed that 12 of the 13 patients monitored after $4-6 \mathrm{~h}$ of treatment initiation improved their serum 
Table 3 Patients and disease characteristics at admission

\begin{tabular}{|c|c|}
\hline Patients, n (\%) & 35 \\
\hline - Male & $16(46)$ \\
\hline - Female & $19(54)$ \\
\hline Median age, years (range) & $66(42-85)$ \\
\hline \multicolumn{2}{|l|}{ Type of cancer, $\mathrm{n}^{\circ}$ of patients } \\
\hline - SCLC & 15 \\
\hline - NSCLC & 7 \\
\hline - Rectal adenocarcinoma & 1 \\
\hline - Ovary & 1 \\
\hline - Breast & 1 \\
\hline - Prostate & 1 \\
\hline - Ampulloma & 1 \\
\hline - Multiple myeloma & 1 \\
\hline - Colon & 1 \\
\hline - Oesophagus & 1 \\
\hline - Refractory diffuse large B-cell Lymphoma & 1 \\
\hline - Thymoma & 1 \\
\hline - Urothelial & 1 \\
\hline - Neuroendocrine & 1 \\
\hline - Unknown primary site & 1 \\
\hline \multicolumn{2}{|c|}{ Vital signs prior tolvaptan treatment, median (range) } \\
\hline - Weight (kg) & $67(52-80)($ from $n=34)$ \\
\hline - Heart rate (bpm) & $\begin{array}{l}85(66-100)(\text { from } n= \\
18)\end{array}$ \\
\hline - Blood pressure (mmHg) & $\begin{array}{l}128(155-110)-72(85- \\
60)(\text { from } n=17)\end{array}$ \\
\hline $\begin{array}{l}\text { Median natremia prior tolvaptan treatment } \\
\text { initiation, } \mathrm{mmol} / \mathrm{L} \text {, (range) }\end{array}$ & $125(117-130)$ \\
\hline \multicolumn{2}{|l|}{ Type of hyponatremia, $\mathrm{n}^{\circ}$ of patients $(\%)$} \\
\hline - Mild (130-135 mmol/L) & $2(6)$ \\
\hline - Moderate (120-129 mmol/L) & $31(88)$ \\
\hline - Severe (Below 120 mmol/L) & $2(6)$ \\
\hline Patient symptomatic, $\mathrm{n}^{\circ}$ of patients $(\%)$ & $26(74)$ \\
\hline - Headache & $13(50)$ \\
\hline - Asthenia & $6(23)$ \\
\hline - Weakness & $4(15)$ \\
\hline - Dizziness & $4(15)$ \\
\hline - Confusion & $3(12)$ \\
\hline - Unsteady gait & $3(12)$ \\
\hline - Disorientation & $2(8)$ \\
\hline - Fall & $1(4)$ \\
\hline - Sleepiness & $1(4)$ \\
\hline $\begin{array}{l}\text { Treatments before Tolvaptan, } \mathrm{n}^{\circ} \text { of patients } \\
\quad(\%)\end{array}$ & $11(31)$ \\
\hline - Fluids restriction & $11(31)$ \\
\hline - Hypertonic infusion & $3(9)$ \\
\hline - No prior treatment & $24(69)$ \\
\hline \multicolumn{2}{|l|}{ Results (Tolvaptan) } \\
\hline \multicolumn{2}{|c|}{ Patients monitored after treatment initiation, $\mathrm{n}^{\circ}$ of patients $(\%)$} \\
\hline - After 4-6h & $13(37)$ \\
\hline - After 24 hours & $35(100)$ \\
\hline
\end{tabular}

Table 3 (continued)

\begin{tabular}{|c|c|c|c|}
\hline Patients, n (\%) & \multicolumn{3}{|l|}{35} \\
\hline \multicolumn{4}{|c|}{ Days to restore the natremia, $\mathrm{n}^{\circ}$ of patients $(\%)$} \\
\hline - 1-4 days & \multicolumn{3}{|c|}{$18(51)$} \\
\hline - More than 4 days & \multicolumn{3}{|c|}{$13(37)$} \\
\hline - Partial response & \multicolumn{3}{|l|}{$3(9)$} \\
\hline - No response & \multicolumn{3}{|l|}{$1(3)$} \\
\hline Adverse events reported, $n^{\circ}$ of patients & Mild & Moderate & Severe \\
\hline - Thirst & 9 & 3 & 0 \\
\hline - Polyuria & 6 & 4 & 0 \\
\hline - Dry mouth & 4 & 0 & 0 \\
\hline - Hepatic enzymes elevation & 2 & 0 & 0 \\
\hline - Others & 0 & 0 & 0 \\
\hline
\end{tabular}

NSCLC no small cell lung cancer, $S C L C$ small cell lung cancer

sodium levels without an overcorrection, with two patients needing a dose escalation to $30 \mathrm{mg}$. After $24 \mathrm{~h}, 2$ of the 35 patients had no serum sodium correction, 1 patient a slight over correction $(13 \mathrm{mmol} / \mathrm{l})$, and all the rest of patients had correct corrections of their serum sodium. The time to restore their natremia was $1-4$ days for 18 patients, more than 4 days (maximum was 29) for 13 patients, a partial response (defined as an improvement of sodium levels without reaching normal values) for 3 patients, and no response (described as no sodium improvement) for 1 patient. Twenty-four patients stopped tolvaptan without any adjustment period when they needed it, 2 had a gradual dose reduction, and 9 reduced the dose by having it on alternate days until withdrawn. Adverse effects were reported in 17 patients, mainly thirst $(9$ mild and 3 moderate) and polyuria (6 mild and 4 moderate). Only four mild cases of dry mouth and two mild cases of hepatic enzymes elevation (both of them with liver metastases) were reported. No cases of osmotic demyelization or other adverse events were reported. Common Terminology Criteria for Adverse Events V4.0 (CTCAE scale) was used to grade the severity of each adverse events. Twenty patients could receive chemotherapy after their serum sodium correction $(9$ immediately after, 1 after $3 \mathrm{~h}, 1$ after $5 \mathrm{~h}, 5$ after $24 \mathrm{~h}$, and 4 after $48 \mathrm{~h}$ ), and 1 of them also underwent radiotherapy after the correction. There was no morbidity nor mortality associated to the use of tolvaptan throughout the treatment. This data reinforces the drug safety described in previous publications $[1,9,31]$. General mortality fluctuated between 4 and 68 weeks since the beginning of hyponatremia treatment $(n=16)$. However, this data is of limited value due to the sample being small and heterogeneous (tumors at different stages of the disease; the tumor is often diagnosed long before the hyponatremia, mortality is thus related to the evolution of the disease itself). 
Based on the findings from these clinical cases, all hyponatremia patients were correctly treated in the DCU, with no serious adverse effects from the medication. They all started chemotherapy in no more than $48 \mathrm{~h}$. Subsequently, the treatment with tolvaptan might have led to an improvement of the ECOG-performance status. In addition, all patients benefited from the effective management of SIADH by receiving the hyponatremia treatment in the same place they received their chemotherapy and also by being able to go home instead of going through the inconveniences and risks that can arise with hospitalization. Our conclusion is that hyponatremia can be treated in hospital DCU as long as proper monitoring of the serum levels can be performed.

Despite the limitations of retrospective observational studies, the positive results of these cases support the hypothesis that tolvaptan can be used effectively and safely in hospital DCU. There is no other similar published data so our experience and recommendations can be a good reference for other professionals in the field who may want to benefit from treating their patients with tolvaptan as outpatients instead of in hospital.

\section{Recommendations for the use of tolvaptan in hospital DCU}

Based on the previous information, some minimum recommendations are proposed.

\section{Patient selection}

- The most suited patients for this protocol are those with moderate hyponatremia $(120-129 \mathrm{mmol} / \mathrm{l})$ with mild/moderate symptoms, currently on anti-cancer active or palliative treatment (provided that the correction of hyponatremia is part of their established treatment strategy).

- Patients at higher risk of demyelination syndromes (those with hypoxia, alcoholism, or malnutrition) should be avoided since they would need closer management [9]. Patients must not present any contraindications for the use of tolvaptan like hypersensitivity to the active substance/excipients, anuria, volume depletion, hypovolemic hyponatremia, and hypernatremia, patients who cannot perceive thirst and pregnancy or breast feeding [9].

- Patients must be able to stay at the hospital within $6 \mathrm{~h}$ for an analytical control and then return again $24 \mathrm{~h}$ after treatment initiation for further controls. In addition, they should live at less than 1-h distance from hospital in case of emergency as well as have good family support. This protocol can only be carried out in DCU with a minimum of $12 \mathrm{~h}$ continued attention so patients can have a control $6 \mathrm{~h}$ after treatment initiation.
- Patients/their relatives will be informed about the possible side effects of the treatment with tolvaptan and asked to return to hospital immediately if the patient experiences any of them.

\section{Treatment dose}

- After establishing the diagnosis and verifying the selection criteria are met, treatment with tolvaptan can be initiated in DCU at a dose of $15 \mathrm{mg}$ once daily. The dose may be increased to a maximum of $60 \mathrm{mg}$ once daily as tolerated to achieve the desired level of serum sodium [9]. Patients may drink fluids freely after treatment initiation [9].

\section{Analytical controls after the first tolvaptan dose}

- An analytical control (for serum sodium and volume status) is recommended no later than $6 \mathrm{~h}$ after. If there is no over rapid serum sodium correction, the patient can be sent home and have another control $24 \mathrm{~h}$ after treatment initiation. If it shows worsening of the patient's condition or serum sodium correction exceeds $6 \mathrm{mmol} / \mathrm{l}$ within $6 \mathrm{~h}$, the patient should be hospitalized for closer monitoring and administration of hypertonic fluids.

- If serum sodium increases $\geq 12 \mathrm{mmol} / \mathrm{l}$ within $24 \mathrm{~h}$ or $\geq 18 \mathrm{mmol} / 1$ within $48 \mathrm{~h}$ or there is a hypernatremia $>145 \mathrm{mmol} / \mathrm{l}$, tolvaptan treatment needs to be interrupted or discontinued followed by administration of hypotonic fluid [9]. If it increases $<136+\leq 5 \mathrm{mmol} / \mathrm{l}$ within $24 \mathrm{~h}$, the tolvaptan dose should be increased.

- Further controls will be done after 3-10 days depending on patients' overall condition and serum sodium correction as well as circumstances like the date of their next appointment.

\section{Treatment duration and withdrawal recommendation}

- Tolvaptan should continue until the underlying disease is adequately treated or until hyponatremia is no longer a clinical problem [10]. According to the results of our series, natremia was normalized in $51 \%$ of patients in the first 4 days, increasing to $88 \%$ of them within 29 days of treatment with tolvaptan.

- For patients on a 15-mg dose, tolvaptan can be withdrawn abruptly or reduced gradually/taken on alternate days, as clinically indicated. If patients are on higher doses, i.e., $30 \mathrm{mg}$, gradual dose reduction is preferred until the dose reaches $15 \mathrm{mg}$ and then it can be withdrawn abruptly or gradually as mentioned before. 
With regard to cancer:

- For SCLC (highly chemo sensible): If there is an improvement of the hyponatremia after 7 days, to reduce the dose to one tablet every $48 \mathrm{~h}$ or $7.5 \mathrm{mg}$ every $24 \mathrm{~h}$; if there is no improvement, to keep the same dose. If there is improvement after 14 days, stop the treatment. Have an additional control after 21 days.

- For head and neck cancer: Since the patient is on radiotherapy, to have him/her controlled every week to consider stopping tolvaptan. If the cancer is disseminated, the management would be similar to that of SCLC.

- For lymphomas: To use a similar management than for SCLC since it is highly chemo sensible.

- Other tumors: It will depend on the treatment outcomes and the treatment schedules.

- In palliative care: Always keep tolvaptan if there is an improvement in the symptoms.

\section{When to start chemotherapy}

Start chemotherapy as soon as possible, depending as well on other factors like the tumor type or the date of the week.

\section{Suggested algorithm for the use of tolvaptan in DCU}

The main mentioned recommendations for the use of tolvaptan in patients with moderate hyponatremias (120-
$129 \mathrm{mmol} / \mathrm{l}$ ) candidate or not candidate for chemotherapy in hospital DCU have been established based on the described clinical experience and publications. The aim of these recommendations is to guide other professionals in the field to consider treating their moderate SIADH-related hyponatremia oncology patients in hospital DCU (Fig. 2).

To summarize, it is suggested to administer $15 \mathrm{mg}$ of tolvaptan at hospital DCU with a control of the natremia $6 \mathrm{~h}$ after. If the control shows worsening or over correction of serum sodium levels, the patient should be hospitalized for closer monitoring. If the patient shows improvement, another control should be performed $18 \mathrm{~h}$ after. If the control shows improvement, chemotherapy can be started after $24 \mathrm{~h}$ in those patients that are candidate for it and withdrawal of tolvaptan will be considered as previously described. In those not candidate to chemotherapy, tolvaptan therapy should be continued until symptoms improve. In all cases, another control of serum sodium levels should be performed 3-10 days after.

\section{Conclusions}

Our retrospective study confirms that tolvaptan can be administered ambulatory in hospital DCU in those patients with a moderate hyponatremia $(120-139 \mathrm{mmol} / \mathrm{l})$ with mild-tomoderate symptoms, currently on anti-cancer active or palliative treatment.

The correction of hyponatremia in cancer patients can help reduce hospital stays, potentially resulting in savings, improve
Fig. 2 Recommendations for treating hyponatremia in cancer patients in hospital day care units

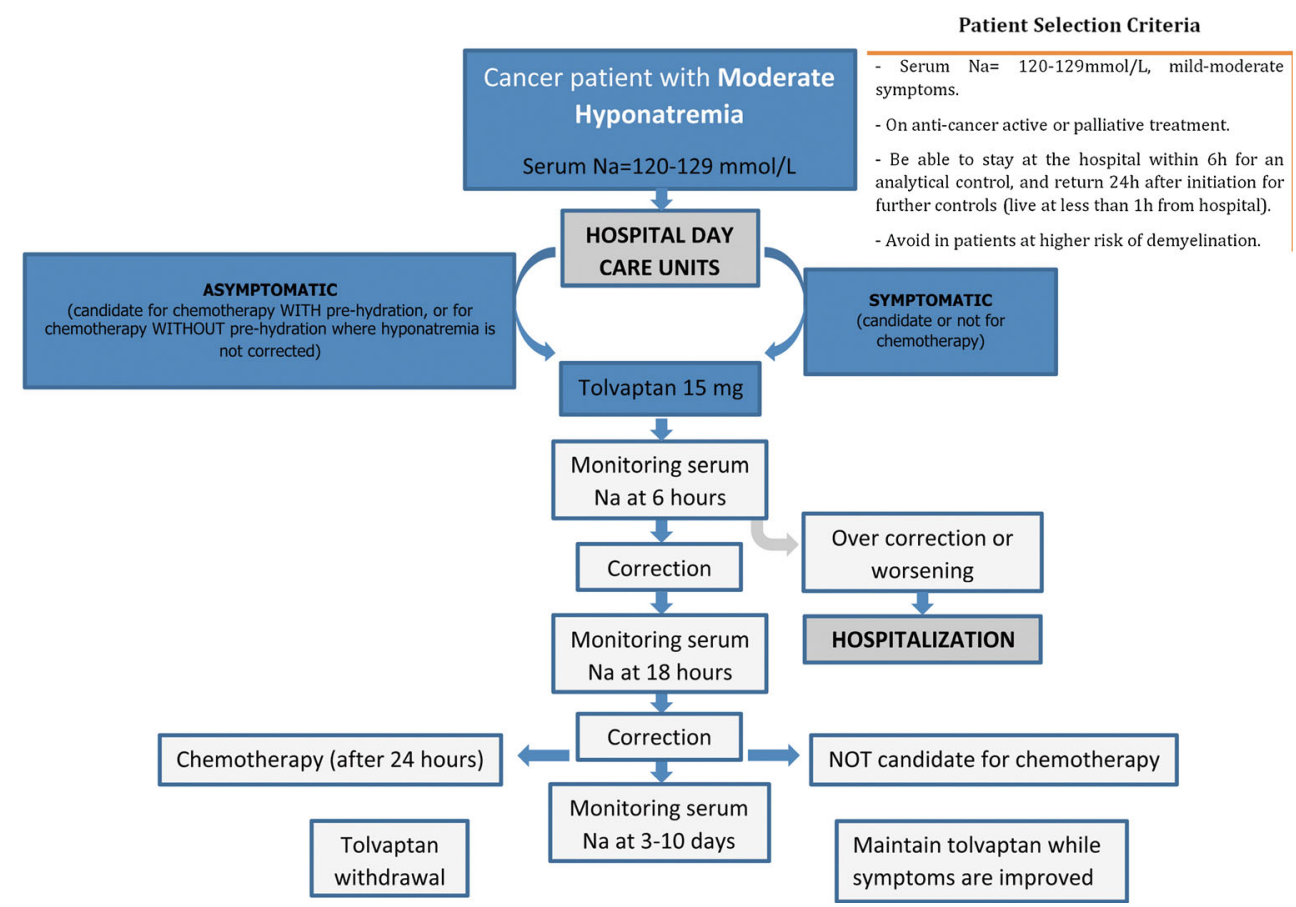


hyponatremia-related symptoms, as well as increase patients overall quality of life and survival.

The use of tolvaptan has proven to be safe and effective in the therapeutic management of SIADH-related hyponatremia in hospital DCU.

Conflict of interest This publication was funded by Otsuka Pharmaceutical Spain, without involving any legal commitment on the results. None of the authors who participated in this paper work or have worked in Otsuka. All authors have collaborated in the conception, drafting, review, and approval of the manuscript submitted, and agreed to allow the journal to review their data if requested.

Open Access This article is distributed under the terms of the Creative Commons Attribution-NonCommercial 4.0 International License (http:// creativecommons.org/licenses/by-nc/4.0/), which permits any noncommercial use, distribution, and reproduction in any medium, provided you give appropriate credit to the original author(s) and the source, provide a link to the Creative Commons license, and indicate if changes were made.

\section{References}

1. Verbalis JG, Adler S, Schrier RW, for the SALT Investigators et al (2011) Efficacy and safety of oral tolvaptan therapy in patients with the syndrome of inappropriate antidiuretic hormone secretion. Eur J Endocrinol 164:725-732

2. Alcázar R, Puerta M (2013) SIADH: Pathophysiology, classification and diagnosis in the hospitalized patient. FMC: Oncología 8: supl 1

3. Runkle I, Villabona C, Navarro A et al (2014) Tratamiento de la hiponatremia secundaria al syndrome de secreción inadecuada de la hormona antidiurética: algoritmo multidisciplinar. Nefrol 4:439450

4. Sørensen JB, Andersen MK, Hansen HH (1995) Syndrome of inappropriate secretion of antidiuretic hormone (SIADH) in malignant disease. J Intern Med 238:97-110

5. Gill G, Huda B, Boyd A et al (2006) Characteristics and mortality of severe hyponatraemia: a hospital based study. Clin Endocrinol 65:246-249

6. Sherlock M, O'Sullivan E, Agha A et al (2006) The incidence and pathophysiology of hyponatraemia after subarachnoid haemorrhage. Clin Endocrinol 64:250-254

7. Sherlock M, O'Sullivan E, Agha A et al (2009) Incidence and pathophysiology of severe hyponatraemia in neurosurgical patients. Postgrad Med J 85:171-175

8. Shea AM, Hammill BG, Curtis LH et al (2008) Medical costs of abnormal serum sodium levels. J A Soc Nephrol 19:764-770

9. Samsca (2014) Summary of product characteristics

10. De las Peñas R, Escobar Y, Henao F et al (2014) SEOM guidelines on hydroelectrolitic disorders. Clin Transl Oncol. doi:10.1007/ s12094-014-1234-2

11. Castillo JJ, Vicent M, Justice E (2012) Diagnosis and management of hyponatremia in cancer patients. Oncologist 17:756-765
12. Ellison DH, Berl T (2007) The syndrome of inappropriate antidiuresis. N Engl J Med 356:2064-2072

13. De las Peñas R (2013) Syndrome of inappropriate antidiuretic hormone secretion in the hospitalized oncologic patient. FMC: Oncología 8: supl 1

14. Schrier RW, Bansal S (2008) Diagnosis and management of hyponatremia in acute illness. Curr Opin Crit Care 14:627-634

15. Adrogue HJ, Madias NE (2000) Hyponatremia. N Eng J Med 342: 1581-1589

16. Verbalis JG, Goldsmith SR, Greenberg A et al (2013) Diagnosis, evaluation, and treatment of hyponatremia: expert panel recommendations. Am J Med 126:S1-S42

17. Hannon MJ, Thompson CJ (2010) The syndrome of inappropriate antidiuretic hormone: prevalence, causes and consequences. Eur J Endocrinol 162:S5-S12

18. Grohé $\mathrm{C}$, et al. Hyponatraemia-SIADH in lung cancer diagnostic and treatment algorithms. Crit Rev Oncol Hematol. 2015 Apr 23. doi: 10.1016/j.critrevonc.2015.04.005

19. Gankam-Kengne F, Ayers C, Khera A et al (2013) Mild hyponatremia is associated with an increased risk of death in an ambulatory setting. Kid Int 83:700-706

20. Hansen O, Sorensen P, Hansen KH (2010) The occurrence of hyponatremia in SCLC and the influence on prognosis. A retrospective study of 453 patients treated in a single institution in a 10-year period. Lung Cancer 68:111-114

21. Berardi R, Caramanti M, Castagnani M et al. (2015) Hyponatremia is a predictor of hospital length and cost of stay and outcome in cancer patients. Support Care Cancer. Mar 5

22. Berardi R, Caramanti M, Fiordoliva I et al (2015) Hyponatraemia is a predictor of clinical outcome for malignant pleural mesothelioma. Support Care Cancer 23:621-626

23. Waikar SS, Mount DB, Curhan GC et al (2009) Mortality after hospitalization with mild, moderate, and severe hyponatremia. Am J Med 122:857-865

24. Doshi SM, Shah P, Lei X et al (2012) Hyponatremia in hospitalized cancer patients and its impact on clinical outcomes. Am J Kidney Dis 59:222-228

25. Wald R, Jaber BL, Price LL et al (2010) Impact of hospitalassociated hyponatremia on selected outcomes. Arch Intern Med 170:294-302

26. Arraras JI, Illarramendi JJ, Viudez A et al (2013) Determinants of patient satisfaction with care in a Spanish oncology day hospital and its relationship with quality of life. Psycho-Oncology 22:2454 2461

27. Sengupta A, Banerjee SN, Biswas NM et al (2013) The incidence of hyponatraemia and its effect on the ECOG performance status among lung cancer patients. J Clin Diagn Res 7:1678-1682

28. Callahan MA (2009) Economic impact of hyponatremia in hospitalized patients: a retrospective cohort study. Postgrad Med 121: 186-191

29. Sheea AM, Hammill BG, Curtis LH et al (2008) Medical costs of abnormal serum sodium levels. J Am Soc Nephrol 19:764-770

30. Boscoe A, Paramore C, Verbalis JG et al (2006) Cost of illness of hyponatremia in the United States. Effect Resour All 4:10

31. Runkle I, Gómez-Hoyos E, Cuesta-Hernández M et al (2015) Hyponatraemia in older patients: a clinical and practical approach. Rev Clin Gerontol 25:31-52 